\title{
MARPE for the treatment and cure of OSAS: Aruba experience
}

\author{
Abdal Hadi Kawaiah', Ananda Kumar Kondepati², Shalini Devaprasad Pasumarthi ${ }^{3}$, \\ Tulika Mishra ${ }^{4}$, Pratik Kumar Singh ${ }^{5}$, Pukhraj Singh Nijhar ${ }^{6}$ \\ ${ }^{1}$ Dr. A Kawaiah, Orthodontics Clinic, Aruba, ${ }^{2}$ Professor and Director, Department of Physiology, Aureus University \\ School of Medicine, Oranjestad, Aruba, Dutch Caribbean, ${ }^{3}$ Professor, Department of Microbiology, Aureus University \\ School of Medicine, Oranjestad, Aruba, Dutch Caribbean, ${ }^{4}$ Assistant Professor, Department of Microbiology, Aureus \\ University School of Medicine, Oranjestad, Aruba, Dutch Caribbean, ${ }^{5.6}$ MBBS Student, Aureus University School of \\ Medicine, Oranjestad, Aruba, Dutch Caribbean
}

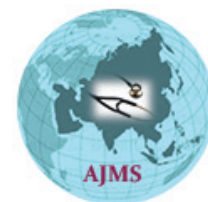

\section{A B S T R A C T}

Obstructive sleep apnea syndrome (OSAS) is defined as "the stoppage of ventilation or incidence of significant hypoventilation during sleep, which is characterized by episodes of partial or complete upper airway obstruction related with hypoxemia and/or hypercarbia. There have been many treatments reported for this syndrome including Mandibular repositioning appliance (MRA), continuous positive airway pressure (CPAP), weight loss, exercise, intraoral appliance therapy, soft tissue procedures, and maxillomandibular advancement (MMA) surgery. Present study is an attempt where three patients of OSAS have been treated with Miniscrew-assisted rapid palatal expansion (MARPE). The results of BMI, AHI index showed the promising effect. Even the use of MARPE has improved the air flow and increased the rapid palatal area.

Key words: Miniscrew-assisted rapid palatal expansion; Sleep disorder; Obstructive sleep apnea syndrome

\section{Access this article online}

Website:

http://nepjol.info/index.php/AJMS DOI: $10.3126 /$ ajms.v11i5.27803

E-ISSN: 2091-0576

P-ISSN: 2467-9100

Copyright (c) 2020 Asian Journal of Medical Sciences

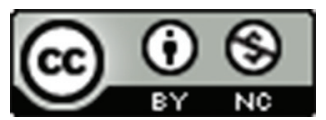

This work is licensed under a Creative Commons Attribution-NonCommercial 4.0 International License.

\section{INTRODUCTION}

Like many other sleep disorders Sleep apnea is a sleep disorder characterized by pauses in breathing or instances of shallow or infrequent breathing during sleep. There are many patients that suffer from sleep apnea. There are manly three forms of sleep apnea, including central sleep apnea (CSA), obstructive sleep apnea (OSA), and complex or mixed sleep apnea. ${ }^{1}$ In CSA, breathing is interrupted by a lack of respiratory effort; in OSA, breathing is interrupted by a physical block to airflow despite respiratory effort, and snoring is common. Obstructive sleep apnea syndrome (OSAS) is defined as "the stoppage of ventilation or incidence of significant hypoventilation during sleep, which is characterized by episodes of partial or complete upper airway obstruction related with hypoxemia and/or hypercarbia". ${ }^{2}$ OSAS is characterized by sleep disturbances resulting in behavioral problems, poor school performance, failure to thrive, daytime fatigue, a slower reaction time, vision problems and in very chronic cases pulmonary hypertension, and core pulmonale. ${ }^{3}$ Leading to many deleterious effects like driving accidents, work related accidents and even to death. Thus, it OSAS demands and early treatment, before it starts affecting the normal life.

The rudimentary origin of OSAS is anatomical obstruction of airway. It can occur in various forms such as large adenoids or chronic mouth breathing which causes the tongue is be unable to mould to the palate and this results in a narrow, high arched palate and poor maxillary growth, which can also result in narrow nasal passages, narrow dental arches and an anterior crossbite, ${ }^{4}$ lingual tonsils 
obstruction, especially in children who are overweight, ${ }^{5}$ and Down syndrome. ${ }^{6}$

To diagnose OSAS is not easy because most of the individuals are not aware of the problem, it is usually noted by others and occasionally suspected by the clinicians. The diagnosis is based upon subjective symptoms along with the formal sleep study through full night polysomnography. ${ }^{7}$ But the places where polysomnography is not available their Oximetry is recommended. ${ }^{8}$ In addition to this Apnea Hypopnea Index (AHI) is considered as diagnostic indicator for sleep apnea. The AHI is the sum of the number of apneas (pauses in breathing) plus the number of hypopneas (periods of shallow breathing) that occur, on average, each hour. To count in the index apneas and hypopneas, collectively called events, must have duration of at least 10 seconds.

OSAS is associated with obesity, cardiovascular problems like high blood pressure, as well as an increased risk of traffic accidents. Some studies have suggested bidirectional relationships between OSAS and diabetes and also with depression. There are various treatment options for OSAS including Mandibular repositioning appliance (MRA), continuous positive airway pressure (CPAP), weight loss, exercise, intraoral appliance therapy, soft tissue procedures, and maxilla mandibular advancement (MMA)surgery. ${ }^{9-11}$ Sleep apnea patients with obesity get to be treated for bariatric surgery, and tracheostomy. In many studies the conventional rapid palatal expansion (RPE) with toothborne anchorage has been suggested asa treatment option for OSAS. ${ }^{12-13}$ But it has been found that RPE is found to be less effective in adult patients, so they are recommended for use of surgery-assisted RPE (SARPE) ${ }^{14-15}$ But SARPE has some disadvantages which includes surgical invasiveness and along retention period. Now a days miniscrew-assisted RPE (MARPE) has been receiving attention by clinicians because of increased orthopedic effects on expansion of the maxillary basal arch width when compared with conventional RPE. ${ }^{16}$ In addition to this it, it induces noninvasive expansion of the mid-palatal suture in adult patients, as contrasting to SARPE. ${ }^{17-18}$

Keeping this in mind the present study was designed to assess the effect of MARPE in three different patients in Aruba who were suffering from OSAS and were restricted with other ailments also.

\section{MATERIALS AND METHODS}

Patient A was 60 years old and was referred to Dr. Kawaiah's clinic office by his Pulmonologist. They could not operate on him because he could not be intubated.
During the exam on Dr. Kawaiah's orthodontic chair, he was not able to be examined with the chair inclined to back. He used to feel choked when the chair is slanting and Dr. Kawaiah used to keep the chair straight. He used to sleep on the chair all the night. He had orthodontic problems like minor crowding, missing teeth, tilted teeth, narrow palate, and well-developed lower turbinate. In addition to that he also has got overbite, Class II malocclusion, Moderate arch discrepancy, insufficient mandibular growth, Severe OSAS, AHI 56, mouth breathing, neck pain and back pain.

Patient $\mathbf{B}$ was a 15-year-old male who started his treatment for OSA due to AHI of $53 / \mathrm{hr}$ in June 2015. His medical history was 4 nose surgeries, adenectomy, FESS and (he underwent) tonsillectomy. Despite all these treatments he still was falling asleep in classroom and not doing good with his grades. He has undergone for bilevel positive airway pressure treatment also. Over the years he went multiple times to check the polysomnography and split night to adjust his pressures and masks as he was growing up. His chief concerns were: Class II Malocclusion, Mouth Breathing, Crossbite (lateral left) deep Impinging, Slight Overjet, Hypertrophy of upper frenum.

Patient $\mathbf{C}$ was a 50 -year-old female with Continuous Positive Airway Pressure (CPAP) treatment since 2016. She was diagnosed with moderate/severe positional OSA. She was snoring since she was a child. But she had tiredness and concentration problems during the day and was falling asleep at work. Her length is $1,53 \mathrm{~m}$ and weight $59 \mathrm{~kg}$ and having a BMI 26.3. She was also suffering of severe depression. For months she was not able to work. The CPAP treatment helped her complains but did not cure her. The ENT specialist operated her for the nose septum deviation and she had tonsillectomy done too.

\section{MARPE}

In this procedure, non-surgical expansion of palatal bone was done with appliances. The procedure of MARPE brings about a combination of basal bone expansion and reduction of stress on the buccal plate. Appliances were fixed in such a way that they generate $88.4 \mathrm{~g} / \mathrm{mm} 2$ of stress at the maximum. The screws used were $11.0 \mathrm{~mm}$ in length and $1.8 \mathrm{~mm}$ in diameter thickness. Screws were fixed in position by bi-cortical penetration. Screws were drill into $2 \mathrm{~mm}$ bone sheath, 1 to $2 \mathrm{~mm}$ air space, $2 \mathrm{~mm}$ soft tissue and 5 to $6 \mathrm{~mm}$ bone engagement. Appliance were placed $1 \mathrm{~mm}$ before the soft palate to get parallel split which was the posterior limit. Based on the bone density, area for anterior placement of the appliance were decided. First pre molar area acts as the landmark in the pattern of expansion. Anteriorly positioned and posteriorly positioned appliances have got different design and fabrication. This 
procedure brings about changes in the airway of the nasal cavity and nasopharynx. The Body Mass Index (BMI) and Apnea-Hypopnea Index (AHI) were checked by standard protocol before and after the treatment.

In addition to that Cone beam computer tomography CBCT was used to evaluate skeletal and dento-alveolar changes in all the three patients using the natural head posture.

\section{RESULTS AND DISCUSSION}

Obstructive sleep apnea (OSA) is an increasingly common, sleep-related breathing disorder. ${ }^{19}$ It is characterized by periodic narrowing and obstruction of the pharyngeal airway during sleep. Untreated OSA is linked with longterm health consequences including cardiovascular disease, metabolic disorders, cognitive impairment, and even the depression. The studies show that most of the people remain undiagnostic may be because of a lack of awareness of the disorder, stigma related to some of the features of the disease such as snoring, access to polysomnography and diagnostic services. ${ }^{20-21} \mathrm{In}$ addition to this if there is no clinical feature related to body mass index than also Clinicians may do not take account of it. It has been found that approximately 20$30 \%$ of patients with a Body mass Index (BMI) greater than 30 and $50 \%$ of those with a BMI greater than 40 have OSA. Continuous positive airway pressure (CPAP) or automatic positive airway pressure (APAP) device has been the most common treatment for the patients with OSAS
The CPAP generates adequate pressure so that the patient's airways open during the sleep. But its uncomfortable for the patient so it affects the prognosis also. Earlier literature suggests that non-surgical treatment with MARPE can be helpful to the patients with OSAS. Mini Implant Assisted Rapid Palatal Expansion, also called as Maxillary Skeletal Expansion, MSE. In this procedure, non-surgical expansion of palatal bone is done with appliances. There are three types of palatal expansions. 1. Tooth borne rapid palatal expansion 2. Bone born rapid palatal expansion and 3 . Tooth and Bone born rapid palatal expansion (MARPE). Although scientific evidence regarding its effects is limited.

The present study was designed to study the impact of MARPE on OSA, which affected the patients AHI and BMI as well.

The Patient A was inserted with MARPE with 6 turns activation right after the insertion. It was tightened by 2 turns/ day for 5 days. In the next round, 1 turn/day for 5 days. Patient improved by $21 \%$ with $5.25 \mathrm{~mm}$ expansion of the maxillo-palatal area. At the end of activation, this patient stopped using CPAP. After 6 months when MARPE was removed the Patient A has shown better aligned teeth (Figure 1), expanded palate, zygomatic bone raised nasal cavity (Figure 2) and all airways expanded (Figures 3 and 4).

The results of other two patients who was also treated with MARPE also followed the similar pattern. Patient B
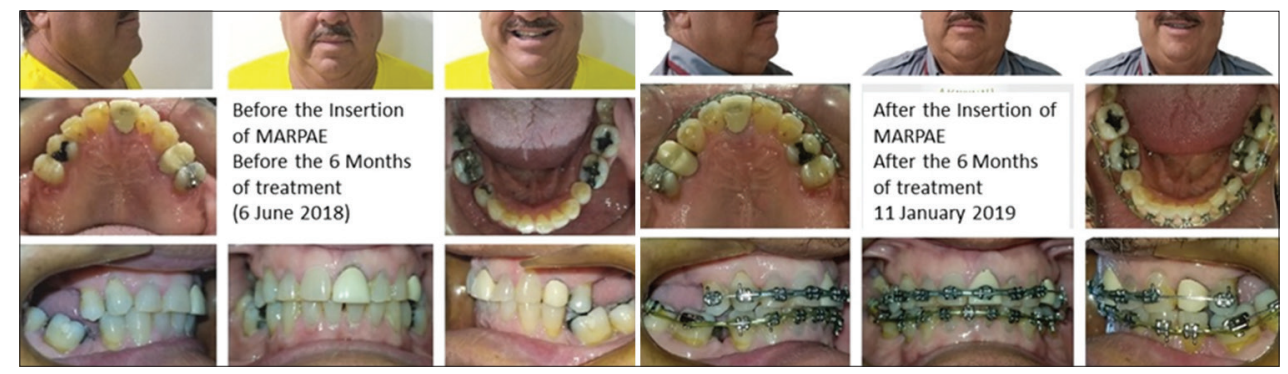

Figure 1: Comparative picture of Patient A before the MARPE Insertion and After Insertion

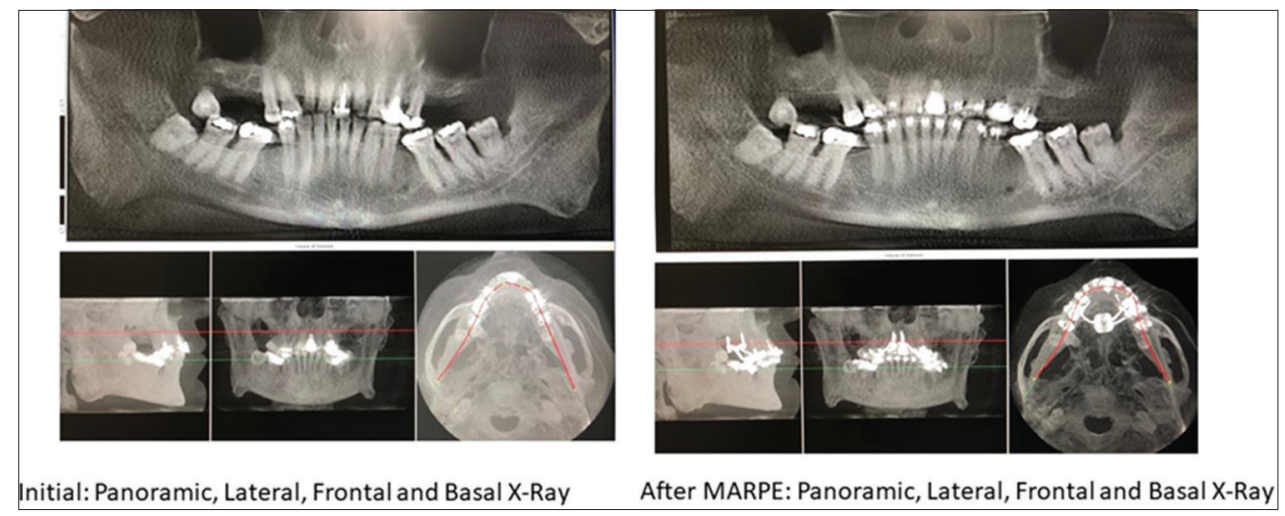

Figure 2: Comparative Panoramic, Lateral, Frontal and Basal $X$ ray of the patient before and after MARPE treatment 


\begin{tabular}{|c|c|c|c|c|c|c|c|}
\hline Patient & Age (Years) & Sex & Weight (Kg) & BMI (Before) & BMI (After) & AHI (Before) & AHI (After) \\
\hline 1 & 60 & $M$ & 115 & 42 & 36.5 & $56 / \mathrm{hr}$ & $22 / \mathrm{hr}$ \\
\hline 2 & 15 & $M$ & 90 & 29 & 25.7 & $53 / \mathrm{hr}$ & $6 / \mathrm{hr}$ \\
\hline 3 & 50 & $\mathrm{~F}$ & 57 & 26 & 24 & $14 / \mathrm{hr}$ & $4 / \mathrm{hr}$ \\
\hline
\end{tabular}

\begin{tabular}{|c|c|c|c|}
\hline PERIOD & PATIENT A & PATIENT B & PATIENT C \\
\hline T0 & $6 / 8 / 2018$ & $9 / 13 / 2018$ & $9 / 26 / 2018$ \\
\hline T0 - Initial Volume & $21.7 \mathrm{CC}$ & $13.7 \mathrm{CC}$ & $17.2 \mathrm{CC}$ \\
\hline T0 - Minimum Area & $97.3 \mathrm{MM} 2$ & 209.7 MM2 & 189.2 MM2 \\
\hline T1 & $8 / 27 / 2018$ & $1 / 4 / 2019$ & $2 / 26 / 2019$ \\
\hline T1 - Initial Volume & $27.9 \mathrm{CC}$ & $17.0 \mathrm{CC}$ & 20.3 CC \\
\hline T1 - Minimum Area & $57.0 \mathrm{MM} 2$ & $251.8 \mathrm{MM} 2$ & 105.8 MM2 \\
\hline T2 & $2 / 25 / 2019$ & $4 / 7 / 2019$ & 4/7/2019 \\
\hline T2 -Initial volume & $21.5 \mathrm{CC}$ & $19.0 \mathrm{CC}$ & $23.0 \mathrm{CC}$ \\
\hline T2 - Minimum Area & $61.4 \mathrm{MM} 2$ & 256.17 MM2 & $185.0 \mathrm{MM} 2$ \\
\hline
\end{tabular}
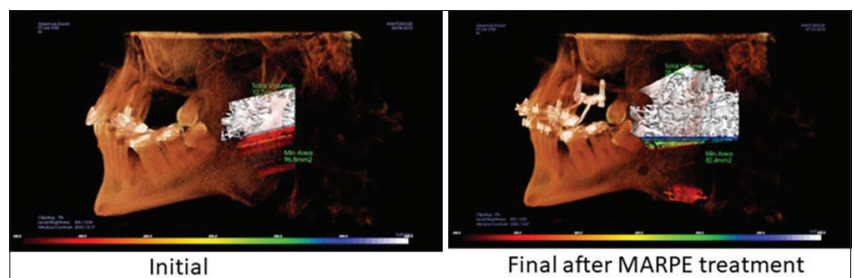

Figure 3: Comparative volume render airway before and after MARPE treatment taken with the cone beam computer tomography

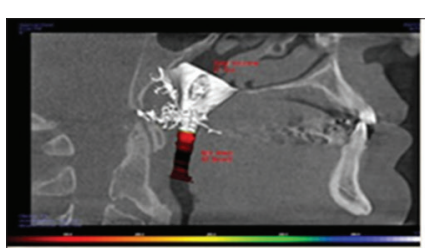

Initials: June 6, 2018

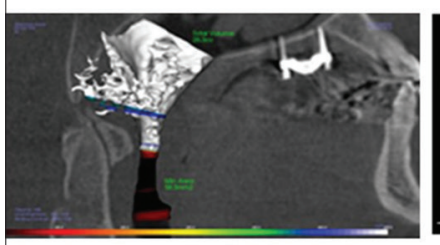

Progress 2: August 27, 2018

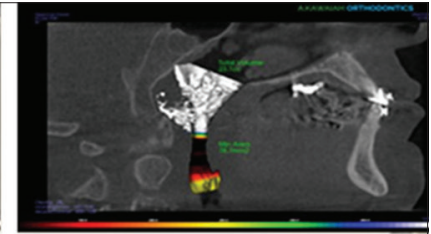

Progress 1: July9, 2018

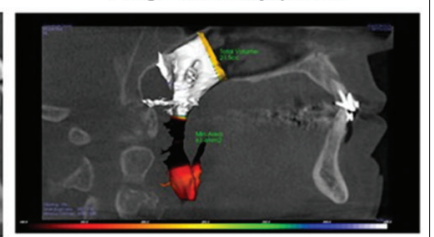

Final Airway
Figure 4: Progress of Airway with MARPE assessed with cone beam tomography

showed maxillo-palatal area increased by $30 \%$ with $6.0 \mathrm{~mm}$ expansion whereas Patient $\mathrm{C}$ showed rise in maxillo-palatal area by $18 \%$ with $6.0 \mathrm{~mm}$ expansion.

The data reveals that rate of Obesity is increasing rapidly and there is a linear correlation of Obesity and OSA. It has been reported that in people with disturbed BMI there are chances that fat deposits in the upper respiratory tract narrowing the airway and there is decrease in muscle activity in that particular region. All these conditions can lead to hypoxic and apneic episodes, ultimately resulting in sleep apnea. ${ }^{22}$ Our data (Table 1) reveal that treatment with MARPE has improved both the BMI and AHI index in our all the three patients, although not much data has been found in reference to MARPE but there are evidence ${ }^{23}$ that supports our result showing that decrease in BMI helps in decreasing the episodes of OSAS.

The results of initial air flow and minimum area as shown in Table 2 also supports the fact that definitely usage of MARPE has improved the condition of OSAS which are in corroboration of earlier findings ${ }^{24}$, Showing that usage of MARPE improves airflow and decreases resistance in Upper Airway.

\section{CONCLUSION}

OSAS, Obstructive sleep apnea disorder, is becoming a growing problem not only for the island of Aruba but also for the rest of the world. This study is an attempt where we can expose to the entire world that using MARPE for patients older than 40 years is seen to be a great solution. Not only does MARPE help with Sleep Apnea, but also helps with symptoms such as: headaches, neck pains, hypertension, diabetes, hypoxemia, dry mouth, and also no more collapsing of the pharynx. This treatment is usually done with orthodontic treatments. There are maxillary and mandibular advancements without surgery. This conjoint technique "MARPE expansion and advancement" relieves the pressure on the pharynx, thereby prevents future collapse. The most expansion found is within the nose cavity because the floor of the nose is related to the palate expansion. The expansion within the mouth will give more room to the tongue which will decrease the choking and snoring of the patient. With this abstract Some cases are treated in Dr. Kawaiah's clinic with the problems related to the nose like nasal-septal deviation and/or polyp and hypertrophy of turbinates and even in some cases of allergic rhinitis. The expansion within the nose cavity relieves and lets past the air easily without any surgery. We can also provide the first evidence with polygraph study on Aruba and polysomnography those patients as young as 15 years and old as 60 years can also be treated and cured from their severe OSAS with this new technique MARPE. 
Currently, there is a retrospective study with the 107 patients who are already finished and busy with the MARPE treatment and we have started a prospective study on the island of Aruba with patients older than 40 years old with MARPE and also selected group of OSAS patients with the treatment of MARPE and we hope by the end of this year and next year to show these wonderful results for the entire medical world and patients.

\section{REFERENCES}

1. Morgenthaler $\mathrm{TI}$, Kagramanov $\mathrm{V}$, Hanak $\mathrm{V}$ and Decker PA. Complex sleep apnea syndrome: is it a unique clinical syndrome? Sleep 2006; 29 (9): 1203-1209.

2. Pi-Chang Lee, Betau Hwang,Wen-Jue Soong and C. C. Laura Meng. The Specific Characteristics in Children with Obstructive Sleep Apnea and Cor Pulmonale. The Scientific World Journal Volume 2012, Article ID 757283.

3. Vidya VS and Sumathi F. Rapid Maxillary Expansion as a Standard Treatment for Obstructive Sleep Apnea Syndrome: A Systematic Review. Journal of Dental and Medical Sciences 2015; 14 (2): 51-55.

4. Sullivan S, Li K and Guilleminault C. Nasal obstruction in children with sleep-disordered breathing. Annals Academy of Medicine Singapore 2008; 37: 645-648.

5. Guimaraes CVA, Kalra M, Donnelly LF, Sally SR, Fitz K, Singla S, et al. The frequency of lingual tonsil enlargement in obese children. American Journal of Roentgenology 2008; 190: 973-975.

6. Phillips DE and Rogers JH. Down's syndrome with lingual tonsil hypertrophy producing sleep apnea. Journal of Laryngology \& Otology 1988; 102: 1054-1055.

7. Clinical practice guideline: diagnosis and management of childhood obstructive sleep apnea syndrome. Pediatrics 2002; 109: 704-12.

8. Brouillette RT, Morielli A, Leimanis A, Waters KA, Luciano R and Ducharme FM. Nocturnal pulse oximetry as an abbreviated testing modality for pediatric obstructive sleep apnea. Pediatrics 2000; 105: 405-412.

9. Ahn HW, Cho IS, Cho KC, Choi JY, Chung JW and Baek SH. Surgical treatment modality for facial esthetics in an obstructive sleep apnea patient with protrusive upper lip and acute nasolabial angle. Angle Orthodontist 2013; 83: 355-363.

10. Epstein LJ, Kristo D, Strollo PJ Jr, Friedman N, Malhotra A Patil SP, et al. Clinical guideline for the evaluation, management and long-term care of obstructive sleep apnea in adults. Journal of Clinical Sleep Medicine 2009; 5: 263-276.

11. Kim T, Kim HH, Hong So, Baek SH, Kim KW, Suh SH, et al. Change in the upper airway of patients with obstructive sleep apnea syndrome using computational fluid dynamics analysis:
Conventional maxillomandibular advancement versus modified maxillomandibular advancement with anterior segmental setback osteotomy. Journal of Craniofacial Surgery 2015; 26: e765-e770.

12. Villa MP, Rizzoli A, Rabasco J, Vitelli O, Pietropaoli N, Cecili M, et al. Rapid maxillary expansion outcomes in treatment of obstructive sleep apnea in children. Sleep Medicine 2015; 16:709-716.

13. Taddei M, Alkhamis N, Tagariello T, D'Alessandro G, Mariucci EM and Piana G. Effects of rapid maxillary expansion and mandibular advancement on upper airways in Marfan's syndrome children: a home sleep study and cephalometric evaluation. Sleep Breath2015; 19:1213-1220.

14. Vinha PP, Faria AC, Xavier SP, Christino M and de MelloFilho FV. Enlargement of the pharynx resulting from surgically assisted rapid maxillary expansion. Journal of Oral Maxillofacial Surgery 2016; 74:369-379.

15. Jaipal PR, Rachala MR, Rajan R, Jhawar DK and Ankush B. Management of adult transverse malocclusion with surgically assisted rapid palatal expansion. Journal of Clinical and Diagnostic Research 2016; 10: ZJ10-ZJ12.

16. Park JJ, Park YC, Lee KJ, Cha JY, Tahk JH and Choi YJ. Skeletal and dentoalveolar changes after mini screw assisted rapid palatal expansion in young adults: A cone-beam computed tomography study. Korean J Orthod 2017; 47: 77-86.

17. Wilmes B, Nienkemper $M$ and Drescher D. Application, and effectiveness of a mini-implant- and tooth borne rapid palatal expansion device: the hybrid hyrax. World J Orthod 2010; 11: 323-330.

18. Choi SH, Shi KK, Cha JY, Park YC and Lee KJ. Nonsurgical miniscrew-assisted rapid maxillary expansion results in acceptable stability in young adults. Angle Orthod 2016; 86: 713-720.

19. Heinzer $R$, Vat $S$, Marques-Vidal $P$, MartiSoler H, Andries D, Tobback N, et al. Prevalence of sleepdisordered breathing in the general population: the HypnoLaus study. Lancet Respiratory Medicine 2015;3(4):310-318.

20. Kapur V, Strohl KP, Redline S, Iber C, O'Connor G and Nieto J. Under diagnosis of sleep apnea syndrome in U.S. communities. Sleep Breath 2002;6(2):49-54.

21. Jaiswal SJ, Owens RL and Malhotra A. Raising awareness about sleep disorders. Lung India 2017; 34(3):262-268.

22. Jehan S, Zizi F, Pandi-Perumal SR, Wall S, Auguste E, Myers AK, et al. Obstructive Sleep Apnea and Obesity: Implications for Public Health. Sleep Medicine Disorder 2017; 1(4).

23. Jehan S, Auguste E, Zizi F, Pandi-Perumal SR, Gupta R, et al. Obstructive Sleep Apnea: Women's Perspective. J Sleep Med Disord 2016; 3(6).

24. Hur JS, Kim HH, Choi JY, Suh SH and Baek SH. Investigation of the Effects of Miniscrew-Assisted Rapid Palatal Expansion on Airflow in the Upper Airway of an Adult Patient With Obstructive Sleep Apnea Syndrome Using Computational Fluid-Structure Interaction Analysis. Korean J Orthod 2017; 47(6):353-364.

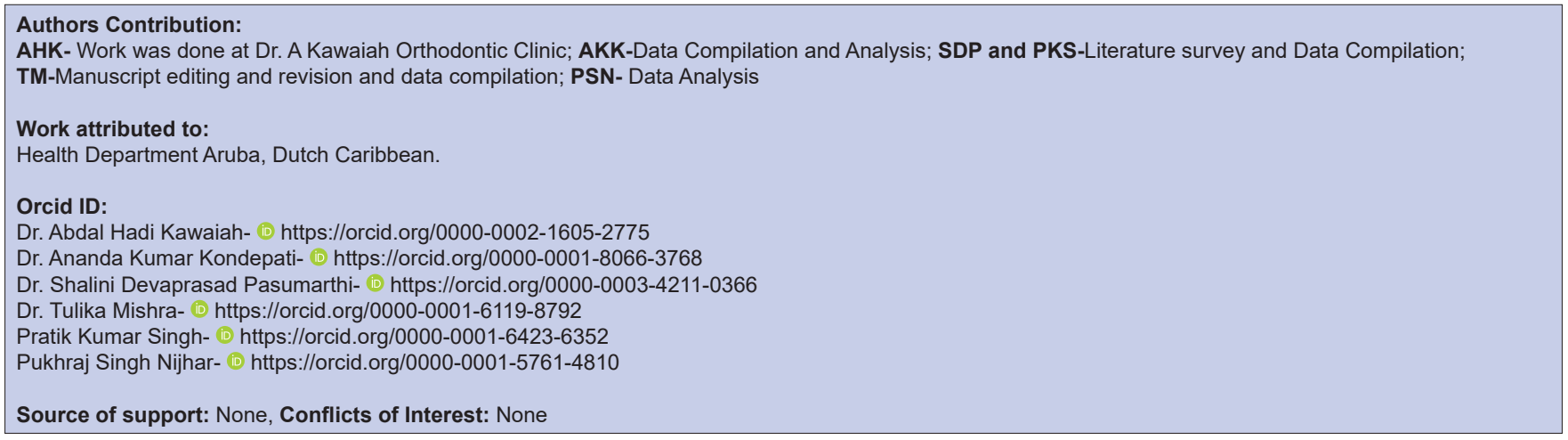

